\title{
Review of: "Engineered miniature CRISPR-Cas system for mammalian genome regulation and editing"
}

\author{
Wenfu Zheng
}

Potential competing interests: The author(s) declared that no potential competing interests exist.

CRISPR-cas systems are powerful in genome engineering. However, the large size of the CRISPR-cas systems (e.g. Cas9 or Cas12a) hinders their wide applications. The development of novel CRISPR-cas systems with a smaller size but a comparable genome editing capability as the currently used CRISPR-cas systems is highly desirable. In this paper, Xu et al. report a miniature Cas system engineered from the type V-F Cas12f system which has 529 amino acids and 62\% and 57\% smaller than the SpCas9 (1368 amino acids) and LbCas12a (1228 amino acids) respectively. This miniature Cas system is below the AAV packaging limit of $4.7 \mathrm{~kb}$. Moreover, it is possible to be easily delivered by nanocarriers such as lipid nanoparticles or gold nanoclusters for a wide range of applications including cell engineering and gene therapy in vitro and in vivo. Totally speaking, this pioneering work may accelerate the popularization of CRISPR-cas technology in the fields of life science and clinical gene therapy. 